\title{
Rethinking transport
}

\section{7-30 April 2020}

Proceedings of 8th Transport Research Arena TRA 2020, April 27-30, 2020, Helsinki, Finland

\section{Detecting possibly threatening conspicuous human behaviour in airport terminals via movement categorization}

\author{
Olaf Milbredt ${ }^{\mathrm{a}, *}$ and Florian Rudolph ${ }^{\mathrm{a}}$ \\ ${ }^{a}$ German Aerospace Center (DLR), Lilienthalplatz 7, 38108 Braunschweig, Germany
}

\begin{abstract}
Facing security issues is one of the most challenging aspects of global transportation. Detecting suspicious behaviour of passengers decreases the risk of an attack inside airport terminals. The preparation of attacks requires deception with concealment. In this paper we describe a methodology to detect categories of passenger movement which are based on analogies to visual and vocal signs of deception. We analyse position data for each passenger which is assumed to be derived by software examining raw surveillance camera data. Passengers are categorized by the shape of their path using physical quantities such as speed. Detected passengers are marked for further inspection. Testing and calibration of the proposed detector is done by a simulation environment covering a medium sized airport terminal building. After calibration, the detector is able to identify all previously defined conspicuous passengers. The approach can be applied to other traffic nodes.
\end{abstract}

Keywords: Human Motion Classification; Trajectory; Variability; Agent-based Simulation

* Corresponding author. Tel.: +49-531-295-2834;

E-mail address: olaf.milbredt(at)dlr.de 


\section{Introduction}

Ensuring transportation to be safe and secure remains a challenging task. The air transportation is heavily exposed to threats, because of the possibly high impact on passengers at the airport, on board an affected flight, and the high range of the subsequent reporting touching the security feeling of people. On one hand, we cannot permit attacks to change the way we live, but on the other hand, this already happened. Almost every attack on the air transportation system led to stronger security measures (cf. Harrison, 2009). Aviation security measures evolved from making security controls mandatory to prevent passengers from carrying firearms through screening of checked baggage to installing full-body scanners.

Recent attacks, such as the bombing in Brussels airport, convey the urge of countermeasures before entering the security control. Video surveillance is used to detect suspicious behaviour in airports. Security officers are required to watch the scenes of up to three displays, so that not every movement can be evaluated. Automatically identifying conspicuous behaviour can draw the attention of an officer to important scenes showing suspicious behaviour. Whether or not a conspicuous behaviour is suspicious depends on many parameters. An experienced security officer can distinguish between these alternatives.

In this paper we describe a methodology to assist security officers to detect conspicuous movement of passengers inside an airport terminal. As in Rowe (2005) we assume that suspicious behaviour shows intentional deceit with concealment. Several non-verbal clues can be employed to reveal deception as characterized by Vrij et al. (2000) and Qin et al. (2004). Rowe (2005) describes analogies between such clues and movement, namely

- Visual: increased blinking and self-grooming $\leftrightarrow$ uncertainty about path direction and speed,

- Vocal: increased hesitation and increased speech errors $\leftrightarrow$ unnecessary stops and starts,

- Vocal: shorter responses and higher voice pitch $\leftrightarrow$ increased speed,

- Verbal: increased over generality and increased irrelevance.

The categories of our approach are based on these analogies. The category fast walking corresponds to the third. The category stopping and waiting corresponds to the second item and may be a preparation of theft as loitering in shopping malls according to Arroyo et al. (2015). The time of waiting can be a hint of unnecessary stopping or intentional waiting. In sight of the security control, stopping and waiting may indicate an attempt to reconnoitre the security control process. The last category walking back and forth is based on the first item and it is an extension of the abrupt direction change used by Aitfares et al. (2016) to detect possible suspicious behaviour. Other intentions may lead to the same ramifications. Additionally, experienced terrorists are trained to disguise their aim. Therefore, this categorization is intended to serve as support for security personnel.

In contrast to expert systems used by Arroyo et al. (2015) or statistical methods used by Rowe (2005) for judging an individual trajectory of a passenger, we use three quantities derived from the spatio-temporal trajectory. This methodology was originally used to categorize the walking behaviour of people inside a shopping mall by Okamoto and Utsumi (2011) in order to provide respective services, such as a layout of the mall or restaurant offers. The methodology uses the velocity vectors which are extracted from positional data.

We built a simulation environment for a medium-sized airport comprising of security control and public area. We apply the methodology to normally and conspicuously moving passengers within the public area. The simulation is conducted with the simulation framework Anylogic (2018). Prescribed path or speed is used to assess the performance of various parameters for the categorization.

Security officers can propose their experience into testing and calibration by refining the movement shape for conspicuous passengers and developing new conspicuous movement shapes. Involving experienced security personnel enhances the quality and acceptance of the categorization. New visualization techniques such as Virtual Reality (VR) can be used as training device for security officers.

With the help of a system empowered by this approach security officers can widen their coverage. Additional to the visual information gathered by security officers themselves, such a system can provide hints where to have a closer look at. Provided that the implementation is not distracting, the system would increase security without the need to increase the number of security officers. 


\section{Related work}

The first generation of video surveillance started in the 1960s. Up to the 1980s Closed Circuit TVs (CCTV) were used with a low level of automation as stated by Räty (2010). During the second generation lasting from the 1980s to 2000 digital CCTVs were used in combination with video processing. In the current third generation from 2000 on a certain grade of automation is achieved. Mostly a similar pattern is used for these systems. This includes the sequential steps (1) foreground objects detection, (2) tracking, and (3) behavioural analysis as stated by Arroyo et al. (2015). This work focuses on (3) behavioural analysis, but relies on (1) and (2).

\subsection{Foreground objects detection}

The field of the first problem, foreground objects detection, has a large number of research results. The choice of an approach for a specific application is difficult, because of varying performance depending on context and scenario. A commonly used technique is background subtraction. Di Stefano et al. (2011) and Brutzer et al. (2011) show different methods using this technique. Maddalena and Petrosino (2008) improve the background subtraction technique by using SOMs (Self-organizing Maps). Such Neural Networks, also known as Kohonen Nets, are used for dimensionality reduction of data by preserving the topology. The authors use a self- organizing map to model background changes and to improve the accuracy of background subtraction.

Reddy et al. (2010) present an approach for improving background subtraction via a new algorithm for estimating he background in cluttered scenes. This is e.g. the case for public places where background initialization without moving objects is almost impossible. The authors use the statistical method Markov Random Field to frame-wise update the background estimation. Verdant et al. (2011) adapted the background subtraction algorithm to image sensors with constrained memory consumption and constrained power consumption. The algorithms proposed use parallel operations per pixel.

Segmentation algorithms based on Gaussian mixture models are used by Stauffer and Grimson (1999) to cope with a changing background. Without re-initialization of the background, errors in the background add over time. Zivkovic (2004) presents an approach using Gaussian mixture with dynamic number of components for each pixel which decreases the computation time. Bouwmans et al. (2008) provide a survey on existing algorithms with Gaussian mixture and extensions for different scenarios as lighting change and moving background.

Yao and Odobez (2007) use a multi-layer approach for background subtraction. Methods employing one layer per pixel, such as colour or intensity, may fail if moving objects have a similar colour to the background. By incorporating more information per pixel the algorithm is able to handle multi-layer background scenes such as addition or removal of stationary items. Zhang and Xu (2006) use fuzzy logic to combine the two layers colour and texture of a scene. Their approach can handle small changes of the background as swaying bushes. El Baf et al. (2008) also use fuzzy logic to combine colour and texture, but employ a different integral for aggregation of fuzzy results. The method was tested on publicly available data for indoor and outdoor surveillance.

\subsection{Tracking}

Tracking moving objects in video surveillance data is an extensive field of research. Different scenarios were considered. Álvarez et al. (2014) present an approach for video surveillance of traffic situations. The approach is able to perform a self-calibration for zooming and is invariant under change of camera placement. Szpak and Tapamo (2011) considers the problem of maritime surveillance. Classical background subtraction algorithms, as stated in Section, are based on the fact that the background is immutable or only slightly changes. The ocean, however, is always in motion. The authors use a level-set approach to overcome this problem. The algorithm produces a contour between background and foreground object which is evolved using a minimization of an energy integral.

Occlusion of detected objects is a challenging task for tracking. Kayumbi et al. (2008) use the combination of multi-camera tracking. The views of all cameras are transformed to a virtual top view of the scene. The authors use graph theory to track detected objects after (partly) occlusion and beyond the borders of a camera view. The 
approach was applied to scenes of American Football. Wu and Nevatia (2007) show an approach for a single camera scenario with partial occlusion.

Human body part detection is a vital approach to overcome the problem of occlusion. Ali and Dailey (2012) consider tracking in high-density crowds. The authors observe human heads for detection and tracking. A further improvement is achieved by combining detection and tracking in one framework. Andriluka et al. (2008) also use one framework for detection and tracking, but additionally consider the movement of the limbs of humans. The accuracy is enhanced by incorporating knowledge about the walking cycle of human beings. Guan and Huang (2015) also uses head detection by modelling each head as ellipse. Beyond improving trajectory information, also contextual information is used.

\subsection{Behavioural analysis}

As core element of a third generation surveillance system and ingredient of a smart surveillance system which is able to automatically raise an alarm, behavioural analysis is an extensive field of research. The research topics include human gesture and posture estimation (see Cristani et al., 2013), biometrics evaluation (cf. Bashir et al., 2008), semantic-based video retrieval (see Patil and Talele, 2015), and cooperative multi camera behavioural analysis (cf. Micheloni et al., 2005; Wang, 2013). Behavioural analysis using head motion is presented by Arsić et al. (2009). Schuller et al. (2008) use face expressions to detect security relevant emotions such as aggressiveness or stress.

This work focuses on the movement behaviour. Yakkali et al. (2018) consider the movement of objects in video surveillance data. Suspicious movement is assumed to occur if a detected object does not move for a number of frames w.r.t. a reference frame. The approach is applied to the identification of unattended baggage or a theft of objects. Arroyo et al. (2015) consider suspicious behaviour in shopping malls such as loitering. Humans are tracked to raise an alarm in the case of an unattended desk with customers. In Aitfares et al. (2016), a method for detecting suspicious movement of humans is presented. In each frame of the camera data, moving objects are identified and the displacement vector with respect to the preceding frame is computed. The mean of this displacement vector is used to judge whether a human moves suspiciously. If the absolute value of the angle exceeds $90^{\circ}$, the individual is marked.

\section{Methodology}

\subsection{Categorization}

Each passenger is grouped into categories, namely

- Fast walking,

- Walking back and forth,

- Stopping and waiting, and

- Normal walking.

The intention of fast walking may lie in the reduction of detection risk or in reaching a flight. Walking back and forth may be driven by nervousness or an expression of boredom. Stopping and waiting may be a hint of spotting or due to waiting for a friend. Only an experienced security officer can differentiate between the reasons. Therefore, this categorization is intended as assistance.

We assume that the trajectory of a passenger is given by a survey system installed, such as camera, LRF, and Wi-Fi. From such systems we derive position data in a certain time interval. From these data it is possible to obtain velocity and speed providing us with a sequence of vectors and numbers.

The approach of Okamoto and Utsumi (2011) is based on the analysis of the history of velocities using the quantities

- Average walking speed,

- Stop-walk ratio, and 
- Variability of the trajectory.

The first quantity is derived by taking the mean over the norm of the velocities. The definition of the second quantity includes a threshold value. Speeds below this value are treated as if the pedestrian stops. Everyone knows this situation. Searching for a location lets us slow down, but not stop entirely. Okamoto and Utsumi (2011) used $0.5 \mathrm{~m} / \mathrm{s}$ as threshold value. The second quantity is then given by the number of speeds below the threshold divided by the number of speeds above the threshold.

The variability is based on the generalization of the variance, the square of the standard deviation, to higher dimensions. This generalization is called covariance matrix. In this case we compute the deviation of the velocities from their mean value taken over all velocities in the sample. Each sample velocity gives us a $3 \times 3$ matrix containing the covariances of each element of the difference with each other element. The variability is then defined as the trace of the arithmetic mean of these matrices.

\subsection{Calibration}

In this section we will show the calibration process for the category walking back and forth. The characteristic of such a movement is a multiple change of the direction of the movement. This behaviour can be capture by the quantity variability. We need to find bounds for the variability to decide whether or not a passenger belongs to this category.

To obtain a feeling for the range of the quantity, we consider a model movement mimicking the behaviour of walking back and forth.
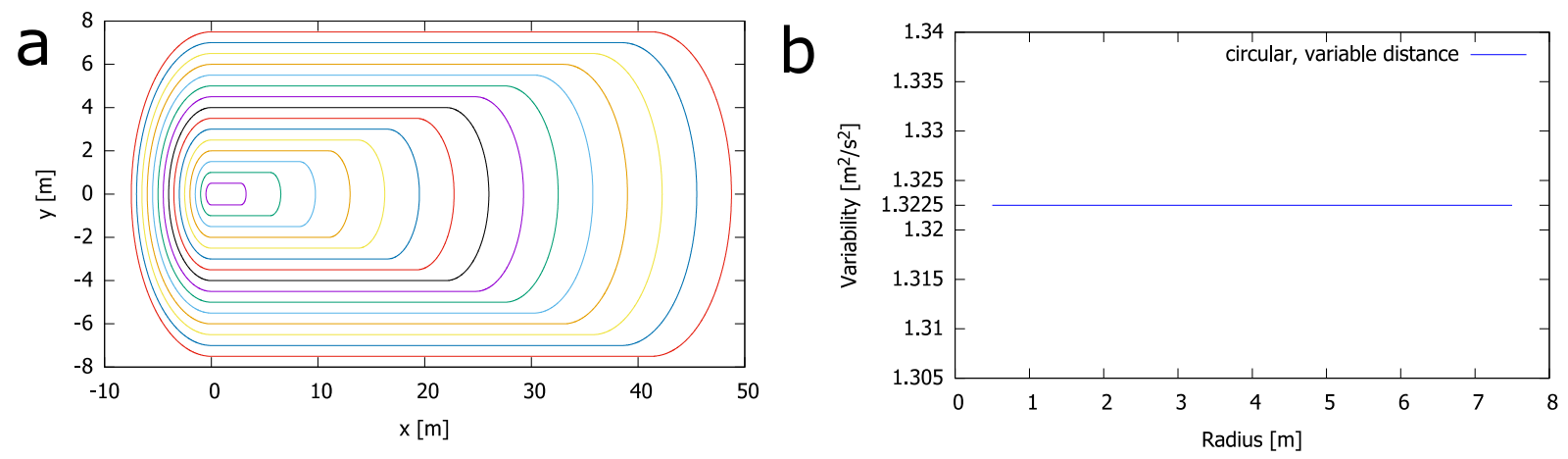

Fig. 1 (a) Paths of a passenger with circular movement; (b) Corresponding variability

Fig. 1a shows the path of a model movement of passengers. The passengers star from the lower left corner and firstly head to the right. The paths lead through a straight and a circular part with various length and radius. The time step is uniformly set to $0.1 \mathrm{~s}$. The speed of all movements is constantly set to $1.15 \mathrm{~m} / \mathrm{s}$. Fig. $1 \mathrm{~b}$ shows the corresponding variability of the movement dependent on the radius of the circular movement. The fraction of radius and distance is chosen to be constant. A varying fraction and a rectangular movement result in the same value of the variability.

From the definition of the variability we derive that it scales with the square of the speed (see Milbredt et al., 2018). We use the value of Fig. $1 \mathrm{~b}$ as base value and scale this value with the square of speed/1.15, where speed denotes the speed of the passenger under consideration. The value obtained is used as a lower bound. Passengers with variability less than this lower bound are not marked as walking back and forth.

\subsection{Simulation}

The pedestrian library of Anylogic (2018) uses the social force model introduced by Helbing and Molnár (1998). The authors assume that a pedestrian acts as if he/she is subject to external forces. The force that influences the desired speed and desired direction of a pedestrian is twofold. Pedestrians try to avoid collisions with obstacles and other pedestrians. This repulsive effect is affected by the private sphere of each pedestrian. A pedestrian 
feels increasingly uncomfortable if he/she comes close to a strange person or an obstacle. The second part of the force describes the behaviour in sight of friends or window displays. This attractive effect leads to the formation of groups. The deviation of the actual velocity is the given by the superposition of the three parts returning to desired speed and desired direction, avoiding obstacles, and advancing to points of interest.

A model of a terminal building shapes the background of the simulation for testing and calibrating. It covers the area of the security control and the area in front serving as transit zone from the check-in desks. The simulation was developed by Alexander Dolt to assess the visual identification of conspicuous passengers. Conspicuously moving passengers as described in Section 3.1 are modelled as follows.

- Fast walking According to Young (1999), the average free-flow walking speed of male pedestrians in an airport is $1.41 \mathrm{~m} / \mathrm{s}$ and the speed of female pedestrians is $1.28 \mathrm{~m} / \mathrm{s}$. The standard deviation is $0.29 \mathrm{~m} / \mathrm{s}$. In the simulation, fast walking passengers have a uniformly distributed speed between $3 \mathrm{~m} / \mathrm{s}$ and $4 \mathrm{~m} / \mathrm{s}$.

- Walking back and forth Passengers of this category follow a pre-defined path letting them circle in the transit zone.

- Stopping and waiting Four areas are defined within he transit area to which passengers of this category advance. The passenger wait for a time uniformly distributed between $5 \mathrm{~s}$ and $30 \mathrm{~s}$.

The position of all passengers is recorded 12.5 times per second, so that the quantities of Section 3.1 can be computed with a small error.

\section{a}

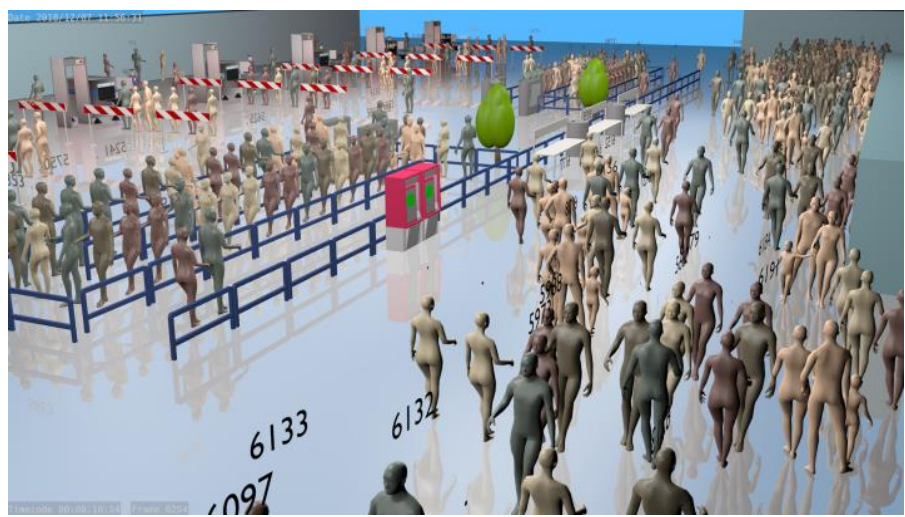

b

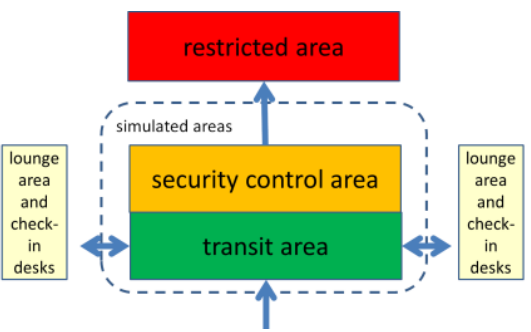

Fig. 2 (a) 3D rendering of a simulation run; (b) Scheme of the areas considered in the simulation

Fig. 2a shows a 3D-rendering of the simulation. It can be seen that the transit area is occupied by a big number of passengers. Some passengers are waiting in a queue to enter the security control. In Fig. 2b, the scheme of the simulation model is depicted. The simulation covers the security control area and the transit zone. The latter connects lounge area and check-in desks in neighbouring buildings on either side with the security control area. At the bottom a direct entrance to the transit zone is located. According to this scheme, Fig. 2a is taken from the bottom left showing the security control area in the upper left and the transit area in the bottom right corner.
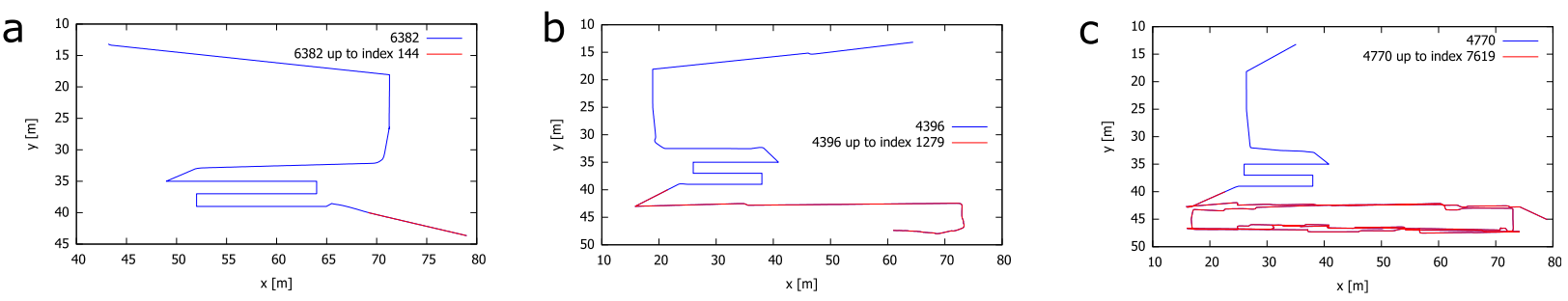

Fig. 3 Paths of passengers: (a) Normal passenger; (b) Passenger walking back and forth by definition; (c) Passenger walking back and forth with characteristic path 


\section{Calculations/Results/Discussion}

We applied our method to the simulation of 1705 passengers during $60.37 \mathrm{~min}$. Each passenger is flagged to be conspicuous or not to be conspicuous. The task of the method is to find conspicuous passengers. The calibration of Section 3.2 is used as a starting point.

Fig. 3 shows the trajectory of three passengers starting in the transit area and moving to the security area through a waiting zone separating both areas. The first passenger, whose trajectory is depicted in Fig. 3a, is marked as normal. He/she proceeds to the waiting zone for the security control without delay. The second passenger is marked as walking back and forth, but does not complete a rectangular movement before entering the security control. The trajectory shown in Fig. $3 \mathrm{c}$ is an example of a passenger walking back and forth within the framework of the simulation. The passenger tries to follow the pre-defined rectangular path.

Due to the snake-like movement of passengers heading to the security control, the variability of their movement is high. Since we know the geometry, we are able to eliminate the part of the trajectory, which does not lie within the transit area. In Fig. 3, the parts of the trajectory lying in the transit area are painted red. The entire path is painted blue.

Table 1. Variability of the entire path and the path lying in the transit area.

\begin{tabular}{lll}
\hline Passenger ID & Variability blue path $\left[\mathrm{m}^{2} / \mathrm{s}^{2}\right]$ & Variability red path $\left[\mathrm{m}^{2} / \mathrm{s}^{2}\right]$ \\
\hline 6382 & 0.272 & 0.018 \\
4396 & 0.441 & 0.476 \\
4770 & 0.240 & 0.257 \\
\hline
\end{tabular}

In Table 1, the variabilities of the paths depicted in Fig. 3 are shown. The variability for the first passenger decreases by one order of magnitude, so that it is easier to detect the normal behaviour. The variabilities of the last two passengers slightly change. The increased variability could stem from the fact that the vertical part and the linear part shown at the top of Fig. 3 is not part of the corresponding red path.
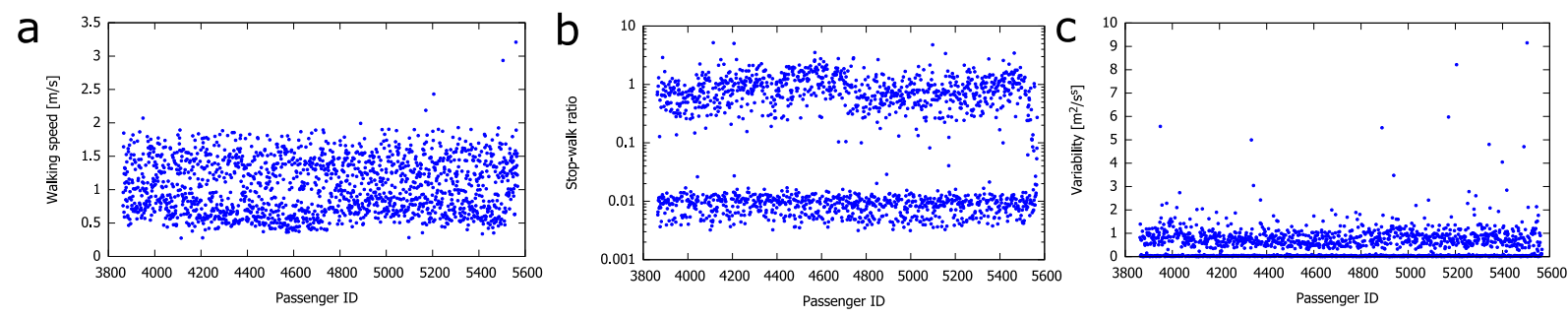

Fig. 4 (a) Walking speed; (b) Stop-walk ratio; (c) Variability of the entire path of all passengers

In Fig. 4, the average walking speed, stop-walk ratio, and variability of all 1705 passengers of the entire path are shown. From Fig. 4a we derive that the average walking speeds of the majority of passengers lies in the range from 0.5 to $2 \mathrm{~m} / \mathrm{s}$. In Fig. $4 \mathrm{~b}$, the scale of the y-axis is logarithmic to provide a better visualization. The threshold used is $0.4 \mathrm{~m} / \mathrm{s}$. The passengers heap about the value 0.01 and 1 . The last picture shows the variability of the entire path. We see that the variability of some passengers is large. 



Fig. 5 (a) Walking speed; (b) Stop-walk ratio; (c) Variability of the path in the transit area of all passengers

In Fig. 5 walking speed, stop-walk ratio, and variability of the path lying within the transit area are shown. It can be seen in Fig. 5a that several fast passengers are present in the data. Although fast passengers are assumed to have a speed greater than $3 \mathrm{~m} / \mathrm{s}$, the average speed is lower, since the passengers start with zero speed. The entrances at both sides and the bottom are modelled as doors so that this behaviour is realistic. Comparing Figs. $4 \mathrm{~b}$ and $5 \mathrm{~b}$ shows that the majority of passengers with higher stop-walk ratio is waiting for the security control so that the heap of passengers in Fig $4 \mathrm{~b}$ about 1 is not present in Fig. 5b. Without the waiting queue for the security control the overall variability decreases as can be derived from Fig. 5c. Nevertheless, the variability of some passengers is greater than $1 \mathrm{~m}^{2} / \mathrm{s}^{2}$.

a

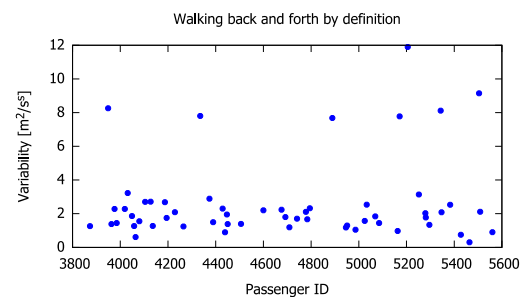

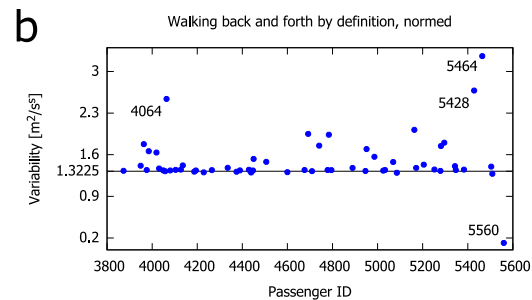

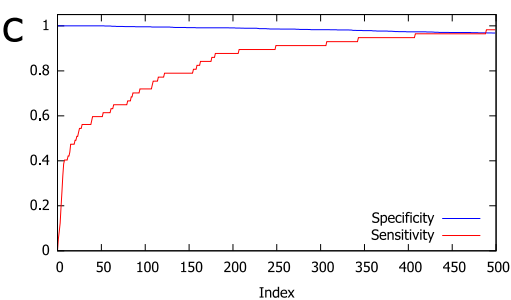

Fig. 6 (a) Variability of passengers belonging to walking back and forth by definition; (b) Scaled variability to match the speed $1.15 \mathrm{~m} / \mathrm{s}$; (c) Sensitivity and specificity of a detector of passengers belonging to walking back and forth dependent on an interval about $1.3225 \mathrm{~m}^{2} / \mathrm{s}^{2}$

We focus on the detection of passengers belonging to the category walking back and forth. Section 3.2 provides a starting point for the detection. Figs. 6a/b show the variability of passengers belonging to the category walking back and forth by definition. Whereas the variabilities of the passengers in Fig. 6 a do not show any characteristic, scaling of the variabilities to match the speed used in Section 3.2 shows a distribution around the value $1.3225 \mathrm{~m}^{2} / \mathrm{s}^{2}$ obtained in Fig. 1b. In Fig. $6 \mathrm{~b}$ we labelled some values which have a big distance to the reference value. On the basis of Fig. $6 \mathrm{~b}$ we implemented a detector for the category walking back and forth by comparing a passenger's scaled variability to the value $1.3225 \mathrm{~m}^{2} / \mathrm{s}^{2}$ obtained in Section 3.2 . Fig. $6 \mathrm{c}$ shows the properties of the detector. A growing interval about the reference value is covered. It grows with growing value of the index in a linear manner approaching the interval [1.27,3.2]. The sensitivity of the detector approaches 0.982 whereas the specificity approaches 0.968 . The specificity leads to 53 false positives. In our data there are 57 passengers of the category walking back and forth present so that only one is not detected. From Fig. $6 \mathrm{~b}$ we derive that Passenger 5560 is not detected. If Passenger 5560 is also included, the number of false positives rises to 198 .
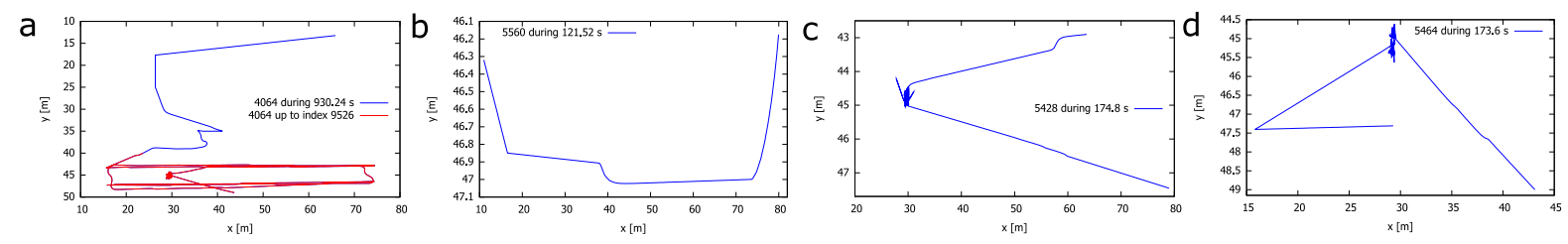

Fig. 7 Trajectory of the four passengers labelled in Fig. 6b

In Fig. 7 we see the trajectories of passengers with high and low normed variability. Fig. 7a shows the trajectory of Passenger 4064. We see the characteristic movement of the category and a part, where the passenger is 
making small movements around the Point $x=30, y=45$. This behaviour belongs to the category stopping and waiting. Since a passenger can have several categories, the behaviour overlaps. The trajectory of Passenger 5560 can be seen in Fig. 7b. From Fig. 6b we derive that this passenger has a low normed variability. The passenger enters the transit area from the left side and exits to the right without the characteristic movement of the category walking back and forth although the simulation marks the passenger as such. The passenger whose trajectories are shown in Figs. 7c/d cannot finish their movement, since they are at the end of the simulation time considered. The high normed variability stems from the fact that these passenger also belong to stopping and waiting. If the passengers of Figs. $7 \mathrm{~b} / \mathrm{c} / \mathrm{d}$ are excluded from the detector, then the number of false positives decreases to 29 .

\section{Conclusions}

Public security is a challenging task affecting the heart of society. In contrast to existing literature, our approach uses trajectory data of pedestrians/passengers collected by installed survey technique to extract possibly securityrelated kinetic behaviour. Against the background of one security officer watching several camera displays, a computer system marking conspicuous behaviour has the potential to increase the awareness.

In this paper we present the categorization of passenger into non-conspicious and several kinds of conspicuous based on their movement. The conspicuous categories are based on analogies stated by Rowe. Whereas the categories fast walking and stopping and waiting represent direct analogies to signs of deception, the last category walking back and forth is an extension. As this category describes the behaviour of repeatedly changing the direction, it is also an extension of Aitfares et al. (2016), who use one direction change of more than 90 degrees to detect suspicious behaviour.

In contrast to Aitfares et al. (2016), our methodology for categorization does not consider the direction change between two video frames, but the shape of the whole trajectory. This property has the advantage that the number of falsely detected passengers is lower. Unlike expert systems used by Arroyo et al. (2015) or statistical methods employed by Rowe (2005), we use three quantities to analyse the trajectory. The categorization is performed by using adjustable bounds for these quantities.

We present the results of virtual experiments showing the performance of the detector for the category walking back and forth. The results show that all previously defined conspicuous passengers are detected with a moderate number of falsely detected passengers. By adapting the bounds it is also possible to detect passengers with one direction change as in Aitfares et al. (2016). The underlying simulation environment can be used to include the experience of security personnel for calibrating the detector and introducing new conspicuous shapes of the trajectory.

Involving security officers increases quality and acceptance. The combination of methodology and simulation enables maintainability and extensibility. Future work will include field tests and the assessment of conspicuous shapes and detector by security experts.

\section{References}

Aitfares, W., Kobbane, A., Kriouile, A., 2016. Suspicious behavior detection of people by monitoring camera, in: 5th International Conference on Multimedia Computing and Systems (ICMCS), pp. 113-117. doi:10.1109/ICMCS.2016.7905601.

Ali, I., Dailey, M.N., 2012. Multiple human tracking in high-density crowds. Image and Vision Computing 30, 966 - 977. doi:10.1016/j.imavis.2012.08.013.

Álvarez, S., Llorca, D., Sotelo, M., 2014. Hierarchical camera auto-calibration for traffic surveillance systems. Expert Systems with Applications 41, 1532 - 1542. doi:10.1016/j.eswa.2013.08.050.

Andriluka, M., Roth, S., Schiele, B., 2008. People-tracking-by-detection and people-detection-by-tracking, in: 2008 IEEE Conference on Computer Vision and Pattern Recognition, pp. 1-8. doi:10.1109/CVPR.2008.4587583.

Anylogic, 2018. Pedestrian simulation. URL: https://www.anylogic.com/airports-stations-shopping-malls/. Accessed December 7th, 2018.

Arroyo, R., Yebes, J.J., Bergasa, L.M., Daza, I.G., Almazán, J., 2015. Expert video-surveillance system for real-time detection of suspicious behaviors in shopping malls. Expert Systems with Applications 42, 7991-8005. doi:10.1016/j.eswa.2015.06.016.

Arsić, D., Hornler, B., Schuller, B., Rigoll, G., 2009. A hierarchical approach for visual suspicious behavior detection in aircrafts, in: $16^{\text {th }}$ International Conference on Digital Signal Processing, pp. 1-7. doi:10.1109/ICDSP.2009.5201258.

Bashir, F., Usher, D., Casaverde, P., Friedman, M., 2008. Video surveillance for biometrics: Long-range multi-biometric system, in: IEEE Fifth International Conference on Advanced Video and Signal Based Surveillance, pp. 175-182. doi:10.1109/AVSS.2008.28.

Bouwmans, T., El Baf, F., Vachon, B., 2008. Background modeling using mixture of gaussians for foreground detection — a survey. Recent Patents on Computer Science 1, 219-237. doi:10.2174/2213275910801030219. 
Brutzer, S., Höferlin, B., Heidemann, G., 2011. Evaluation of background subtraction techniques for video surveillance, in: CVPR 2011, pp. 1937-1944. doi:10.1109/CVPR.2011.5995508.

Cristani, M., Raghavendra, R., Bue, A.D., Murino, V., 2013. Human behavior analysis in video surveillance: A social signal processing perspective. Neurocomputing 100, 86 - 97. doi:10.1016/j.neucom.2011.12.038. special issue: Behaviours in video.

Di Stefano, L., Regazzoni, C., Schonfeld, D., 2011. Advanced video-based surveillance. EURASIP Journal on Image and Video Processing doi:10.1155/2011/857084.

El Baf, F., Bouwmans, T., Vachon, B., 2008. Fuzzy integral for moving object detection, in: IEEE International Conference on Fuzzy Systems (IEEE World Congress on Computational Intelligence), pp. 1729-1736. doi:10.1109/FUZZY.2008.4630604.

Guan, Y., Huang, Y., 2015. Multi-pose human head detection and tracking boosted by efficient human head validation using ellipse detection. Engineering Applications of Artificial Intelligence 37, 181 - 193. doi:10.1016/j.engappai.2014.08.004.

Harrison, J., 2009. International Aviation and Terrorism. Taylor \& Francis, London. doi:10.4324/9780203878712.

Helbing, D., Molnár, P., 1998. Social force model for pedestrian dynamics. Physical Review E 51, $4282-4286$. doi:10.1103/PhysRevE.51.4282.

Kayumbi, G., Mazzeo, P.L., Spagnolo, P., Taj, M., Cavallaro, A., 2008. Distributed visual sensing for virtual top-view trajectory generation in football videos, in: Proceedings of the 2008 International Conference on Content-based Image and Video Retrieval, ACM, New York, NY, USA. pp. 535-542. doi:10.1145/1386352.1386420.

Maddalena, L., Petrosino, A., 2008. A self-organizing approach to background subtraction for visual surveillance applications. IEEE Transactions on Image Processing 17, 1168-1177. doi:10.1109/TIP.2008.924285.

Micheloni, C., Foresti, G.L., Snidaro, L., 2005. A network of co-operative cameras for visual surveillance. IEE Proceedings - Vision, Image and Signal Processing 152, 205-212. doi:10.1049/ip-vis:20041256.

Milbredt, O., Classen, A.B., Grunewald, E., 2018. Classification of air transport passengers' kinetic behavior, in: AIAA Modeling and Simulation Technologies Conference, 8-12 January, Kissimmee, Florida, USA, American Institute of Aeronautics and Astronautics. doi:10.2514/6.2018-0422.

Okamoto, K., Utsumi, A., 2011. Classification of pedestrian behavior in a shopping mall based on LRF and camera observations., in: MVA2011 IAPR Conference on Machine Vision Applications, June 13-15, Nara, Japan. URL: http://www.mvaorg.jp/Proceedings/2011CD/papers/01-01.pdf. Accessed December 11th, 2018.

Patil, S., Talele, K., 2015. Suspicious movement detection and tracking based on color histogram, in: 2015 International Conference on Communication, Information Computing Technology (ICCICT), pp. 1-6. doi:10.1109/ICCICT.2015.7045698.

Qin, T., Burgoon, J., Jr, J., 2004. An exploratory study on promising cues in deception detection and application of decision tree. doi:10.1109/HICSS.2004.1265083.

Räty, T.D., 2010. Survey on contemporary remote surveillance systems for public safety. IEEE Transactions on Systems, Man, and Cybernetics, Part C (Applications and Reviews) 40, 493-515. doi:10.1109/TSMCC.2010.2042446.

Reddy, V., Sanderson, C., Lovell, B.C., 2010. A low-complexity algorithm for static background estimation from cluttered image sequences in surveillance contexts. EURASIP Journal on Image and Video Processing doi:10.1155/2011/164956.

Rowe, N., 2005. Detecting suspicious behavior from positional information URL: https://www.researchgate.net/publication/254898609_Detecting_Suspicious_Behavior_from_Positional_Information. Accessed November 3rd, 2019.

Schuller, B.W., Wimmer, M., Arsić, D., Moosmayr, T., Rigoll, G., 2008. Detection of security related affect and behaviour in passenger transport, in: INTERSPEECH.

Stauffer, C., Grimson, W.E.L., 1999. Adaptive background mixture models for real-time tracking, in: Proceedings. IEEE Computer Society Conference on Computer Vision and Pattern Recognition (Cat. No PR00149), pp. 246-252. doi:10.1109/CVPR.1999.784637.

Szpak, Z.L., Tapamo, J.R., 2011. Maritime surveillance: Tracking ships inside a dynamic background using a fast level-set. Expert Systems with Applications 38, 6669 - 6680. doi:10.1016/j.eswa.2010.11.068.

Verdant, A., Villard, P., Dupret, A., Mathias, H., 2011. Three novell analog-domain algorithms for motion detection in video surveillance. EURASIP Journal on Image and Video Processing 2011, 698914. doi:10.1155/2011/698914.

Vrij, A., Edward, K., Roberts, K.P., Bull, R., 2000. Detecting deceit via analysis of verbal and nonverbal behavior. Journal of Nonverbal Behavior 24, 239-263. doi:10.1023/A:1006610329284.

Wang, X., 2013. Intelligent multi-camera video surveillance: A review. Pattern Recognition Letters 34 , 3 - 19. doi:10.1016/j.patrec.2012.07.005. extracting Semantics from Multi-Spectrum Video.

Wu, B., Nevatia, R., 2007. Detection and tracking of multiple, partially occluded humans by bayesian combination of edgelet based part detectors. International Journal of Computer Vision 75, 247-266. doi:10.1007/s11263-006-0027-7.

Yakkali, T.R., Nayar, R., Sreedevi, I., 2018. Object tracking and suspicious activity identification during occlusion. International Journal of Computer Applications 179, 29-34. doi:10.5120/ijca2018916117.

Yao, J., Odobez, J., 2007. Multi-layer background subtraction based on color and texture, in: 2007 IEEE Conference on Computer Vision and Pattern Recognition, pp. 1-8. doi:10.1109/CVPR.2007.383497.

Young, S., 1999. Evaluation of pedestrian walking speeds in airport terminals. Transportation Research Record: Journal of the Transportation Research Board , 20-26doi:10.3141/1674-03.

Zhang, H., Xu, D., 2006. Fusing color and texture features for background model, in: Wang, L., Jiao, L., Shi, G., Li, X., Liu, J. (Eds.), Fuzzy Systems and Knowledge Discovery, Springer Berlin Heidelberg, Berlin, Heidelberg. pp. 887-893. doi:10.1007/11881599_110.

Zivkovic, Z., 2004. Improved adaptive gaussian mixture model for background subtraction, in: Proceedings of the 17th International Conference on Pattern Recognition (ICPR), pp. 28-31. doi:10.1109/ICPR.2004.1333992. 